\title{
THE PATHOLOGICAL ANATOMY
}

\author{
or \\ C A N I N E "C H O R E A."” \\ $\mathbf{B Y}$
}

W. R. GOWERS, M.D.,

ASSISTANT-PROFFGSOR OF OLINIOAL MFDIOINE IN UNIVBRSITY COLLFGR,

\ND

H. R. O. SANKEY, M.R.C.S.,

RESIDENT MFDICAL OFFICRR TO THR NATIONAL HOSPITAL FOR THE PARALYSED AND RPILEPTIC.

(Received February 13th-Read March 13th, 1877.)

THE two cases which form the subject of this communication were examples of the disease of the dog which is known as " chorea," but which differs somewhat in its characters from the chorea of man. Its chief feature is the occurrence of quick contractions in isolated muscles or groups of muscles, the contractions being separated by considerable intervals, and resembling those in the affection which has been described as "chorea electrica." There is an absence of the spontaneous fidgetty movements and inco-ordination, which are so characteristic of chorea as it is usually seen in man.

In the two cases the movements were similar in character, but differed in extent and degree. In the slighter case they were limited to one fore leg; in the 
other they were widely distributed, affecting all four limbs as well as the muscles of the head and neck. In each animal the effect of section of the cervical spinal cord was observed; in the former the movements persisted for a short time, in the latter they ceased at once. In the former only slight morbid changes were discovered in the nerve-centres; in the latter the cerebellum, spinal cord, and to a less extent the medulla oblongata, were the seat of extensive and unusual alterations.

It may be convenient to describe first the case in which the symptoms and morbid appearances were most marked.

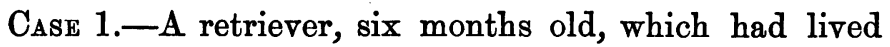
chiefly in a dwelling house, was well until, at four months old, it had a mild attack of distemper. When it had nearly recovered from the distemper, slight twitching was observed in the left foreleg. This increased, and extended to the hind legs, which became affected at the same time, and nearly to the same extent. The right foreleg was then also attacked, and the twitching soon became more severe in it than in the hind legs. Soon all the muscles of the trunk and neck were involved, including the extrinsic laryngeal and the lingual muscles. Those of the eyeballs were free. Weakness in the limbs was then observed, noticed first in the hind limbs; the animal became unable to walk, and on an attempt to do so the body was curved, with the convexity to the right. These symptoms continued without abatement.

When it came under our observation, two months after the commencement of the affection, no change in temper was observed, and there was little evidence of pain. The animal was restless, frequently changing its posture. The muscular twitchings were then general; the contractions quick and sudden, like those caused by the interrupted voltaic current. The muscles of the same part contracted together, e.g., those of the hind legs and tail, but the spasm of the hind legs and of the forelegs was not always simultaneous. The contractions occurred in the same muscles six or 
seven times in a minute; usually there were two or three in rapid succession, and then a long pause. They were more marked while the animal was at rest than when a movement was attempted. The weakness of the limbs, especially of the hind limbs, was very considerable. The dog could just stand, but on attempting to walk the hind legs gave way, and it fell. When lying it was able to move them freely. The tail could be moved with vigour. All the muscles of the right side seemed weaker than those of the left, and thus the curve of the trunk was produced. There was no obvious weakness of the muscles of the head or jaw.

Sensation was considerably diminished in the posterior half of the body. A pin could be driven in without causing the animal to cry or to wake from sleep. Pinching the tail was unfelt. Sensation in the fore legs seemed lessened, but not abolished. A prick caused the animal to look at the part, but not to withdraw the leg. Sensation seemed normal about the shoulders, the neck and the head. The animal had a habit of nibbling at the fore legs, as if they were the seat of some disordered sensation.

There was no affection of the organs of special sense. There was no paralysis of the sphincters.

The dog was placed under the influence of ether and the twitchings completely ceased. When it was allowed to recover partially from the influence of the ether the twitchings recommenced. The spinal cord was afterwards divided below the occiput, and artificial respiration performed by means of a tube previously introduced into the trachea. As soon as the dog had recovered from the effects of the ether, twitchings recommenced in the jaw and the muscles of the face and head, including the external laryngeal muscles, but there were no twitchings in any parts supplied from the spinal cord below the section during the four or five minutes which elapsed before the animal was killed. (The interpretation to be placed on this fact will be considered presently.) 
A careful post-mortem examination revealed no disease of internal organs. No entozoa were found. The heart was healthy. The muscles to the naked eye and the microscope appeared perfectly healthy. The nerve centres were examined in a recent state and after hardening.

No lesion was discovered in the cerebral hemispheres or the central ganglia. On the other hand the spinal cord, the medulla oblongata, and the cerebellum presented extensive alteration.

Examination of the spinal cord in a fresh state (immediately after death) revealed little change. No areas of congestion or ecchymosis were visible. The white substance appeared normal in consistence and tint. Nervecells from the anterior cornua appeared somewhat swollen and unduly granular, but contained no pigment.

After hardening, however, extensive lesions were apparent. The most conspicuous change was the infiltration of limited areas with small round "lymphoid" cells, which varied in size from $\frac{1}{3000}$ to $\frac{1}{4000}$ inch, the average being $\frac{1}{3500}(\cdot 0075 \mathrm{~mm}$.). In most of these cells no nucleus was visible, but in some a large round nucleus occupied about half or two thirds of the area of the cell. These cells were not uniformly infiltrated in the affected areas. In the white substance they were aggregated in tracts, often branching, and extending through part or the whole of the affected column (Pl. XII, figs. 1, 2, 3, and 4). When the infiltration affected the grey substance it was more uniform, but here also the corpuscles were aggregated in masses, as well as scattered. The aggregations in both grey and white matter corresponded to the course of vessels, or of the larger connective-tissue septa in which the vessels run. Whenever a section divided such a tract transversely a vessel could always be detected in its centre (Pl. XII, fig. 5). Where the infiltration was extensive the corpuscles were so densely massed that the individual cells could scarcely be distinguished (Pl. XII, fig. 7), but where it was slighter a vessel could often be seen to be encrusted with a layer of such cells. In some 
places a few only lay in the perivascular sheath, but in other places other similar corpuscles lay outside the perivascular sheath and in the adjacent tissue. In the white substance in the neighbourhood of these tracts similar cells lay among the nerve-fibres, and in some places were so numerous as to occupy a larger area than the nerve-fibres (Pl. XII, fig. 7). In some places, indeed, the nerve-elements appeared to have been destroyed.

These cells were deeply stained with carmine, and still more deeply by logwood. They resembled closely white blood-corpuscles in size and appearance. (The measurements given above correspond closely to those of white blood-corpuscles in hardened tissues.) They were identical in appearance with the white blood-corpuscles which were seen here and there among the blood-discs within the vessels. In some sections the walls of the vessels appeared to contain similar cells, as if in the process of diapedesis. From these appearances we are inclined to regard these cells as being actually white blood-corpuscles, and the morbid change as a leucocytal infiltration.

In some parts, in which the cells were scattered among the nerve-fibres, some were no longer round, but were oval and even angular (Pl. XII, fig. 5), and from the angles of some of these, minute processes extended, while in the neighbourhood were other angular cells, the branches of which extended between the nerve-fibres. These were larger and far more abundant than any existing in the normal cord, and from the transitional forms they appeared to be in the process of development from the infiltrating corpuscles.

In many places near this infiltration there appeared to be an increase in the nuclei of the neuroglia, the interstices between the fibres being studded with minute nuclei, smaller than red blood-discs, and much more numerous than in health. In some parts this was noticeable apart from any leucocytal infiltration.

Where the infiltration had taken place into the grey 
substance, the matrix of the grey matter had undergone a granular degeneration, or else presented an œdematous appearance, its constituent elements being separated by clear spaces, which gave it a spongy appearance.

The nerve-cells in many parts of the cord appeared perfectly healthy. In most places, however, they presented the same granular appearance which was noticed in the fresh state. Where the grey matter was invaded by the leucocytes, the nerve-cells were completely surrounded by them. Some of the cells had a remarkable appearance, their outer boundary was not well defined, the processes had disappeared, the substance of the cell was granular, the nucleus distinct, the cell-substance in the immediate vicinity of the nucleus stained deeply with carmine, that near the outer part of the cell much less deeply, and the indistinctness of the outer boundary gave the cell the appearance as if it were being dissolved (Pl. XIII, figs. 10 and 11). Cells presenting such an appearance were seen very near to cells which had fairly healthy characters, and the change cannot, therefore, be ascribed to any effect of the hardening agent.

In many parts of the cord the substance of the large nerve-cells was encroached upon by rounded vacuoles of various size (Pl. XIII, figs. 8 and 9). Sometimes these lay entirely within the body of the cell, but more frequently they were partly outside in some coagulable fluid in which they had apparently formed in the process of hardening. They were precisely similar to the vacuoles seen in the substance of albuminous exudations in other organs, as into the tubules of the kidney in acute Bright's disease. Many cells were almost destroyed by them. They are probably of pathological significance, even though of artificial origin, and may indicate the presence, in the vicinity of the cells, of the results of excessive action.

Anatomical description of lesions in the spinal cord.Midway between the first and second pair of cervical nerves an extensive infiltration of cells has occurred into the 
right posterior cornu in the position of the posterior vesicular column; the matrix of the nerve-tissue here is granular and broken up; the cells are scattered irregularly through it, and some are aggregated around a vessel. On the left side of the cord, vessels here and there were varicose. A few sections lower down similar infiltration and disintegration exist in the left cervix cornu posterioris, and in addition to the lymphoid cells, blood discs are infiltrated into the tissue.

At the level of the second cervical nerves lymphoid cells are scattered through the left anterior cornu, near the grey commissure, and are aggregated especially around a vessel in this position. In both right and left lateral columns is a scattered infiltration of cells, those on the left side occupying areas of considerable extent. There is no general thickening of the neuroglia.

Third cervical nerves.-Extensive cellular infiltration into the posterior column and into both lateral columns. The anterior columns are perfectly free. In the right lateral column the infiltration is confined to the anterior half, the posterior half being quite free. In the posterior columns the infiltration is in places very dense, and the individual cells cannot always be recognised in the thickened tracts which extend through the white substance. In some places these appear as if consisting of fibrous tissue, but on the margins of these tracts the separate round cells are visible. In many places the perivascular sheaths of the vessels are seen to be distended with these cells. In other places vessels running transversely appear covered with a cellular incrustation. In the lateral columns, besides this cellular infiltration, there is a considerable increase in the minute nuclei between the nervefibres. The left caput cornu posterioris is also infiltrated with lymphoid cells, and a similar infiltration extends along the posterior nerve-roots to the surface of the cord. The pia mater is here and there separated from the surface of the cord by a structureless exudation.

Fifth cervical nerves.-The left anterior cornu appears 
to be literally crammed with leucocytes throughout its anterior half, and from it tracts of similar cells extend along the connective-tissue trabeculø into the adjacent anterior column. Small areas of granular débris exist here and there in the anterior cornu. In the anterior column adjacent to the cellular infiltration there is much increase in the nuclei of the neuroglia, and this increase, in a slighter degree, is to be noticed throughout all the white columns.

Sixth cervical nerves. - The right anterior cornu is here full of leucocytes, and is distinctly enlarged by their infiltration. Its width is just one third more than the left anterior cornu. The infiltration is especially dense in the superficial layers of the grey matter near the white column, and also in the adjacent white substance, and the vessels which pass from the surface through the anterior column are surrounded by cells. The central part of the anterior cornu appears as if in a state of œdema. Instead of a finely granular matrix it has a spongy appearance; there is a network of fine fibrillæ, but these are separated by clear spaces. In the anterior part the large nerve-cells appear swollen aud somewhat granular, and are surrounded by leucocytes. The same appearance of œdema may be seen in the adjacent white column; clear spaces exist between the trabeculø of connective tissue, spaces in which no nerve-fibres or axis-cylinders can be seen.

First dorsal nerves.-The central canal of the cord was widely distended by granular exudation. The posterior columns were densely infiltrated with lymphoid cells, especially the outer half of the right posterior column. In the superficial layers of the posterior columns there is a considerable increase in the neuroglial tissue, almost all the axis cylinders having disappeared, and a nucleated connective tissue remaining in this place.

Second dorsal nerves. - The right half of the cord is fairly healthy, but the left presents extensive changes. The anterior column and the adjacent part of the lateral column present a dense cellular infiltration, and there is also 
some infiltration of the grey substance of the anterior cornu.

Third dorsal nerves.-The cord is fairly healthy. A small number of lymphoid cells lie along the vessels in the lateral column near the right posterior cornu, and the vessels of the grey substance of the posterior cornu have the appearance of the walls being crammed with similar cells.

Fourth dorsal nerves. - The left anterior column, between the anterior cornu and the surface, presents numerous tracts of cellular infiltration. Towards the surface these are very dense, and occupy the whole tissue. The nervefibres in the anterior column are quite distinct, but towards the lateral column they become separated by the infiltration of lymphoid cells, and finally the nerve-fibres disappear, and are replaced by irregular masses of myelin, partly fused together and lying between the dense tracts of cells ; here and there an axis cylinder can still be seen. In several other spots in the neighbourhood of the infiltration the nerve-fibres have disappeared and have left large spaces. The adjacent part of the anterior cornu is crammed with cells in its entire width, and one or two nerve-cells are granular and swollen so as to be globular in form, with very indistinct processes. They appear as if in process of disintegration, as already described.

Fifth dorsal nerves.-The cord presents scarcely any deviation from the natural state, the only change being the presence of tracts of lymphoid cells along the vessels in the posterior column.

Eighth dorsal nerves.-The cellular infiltration is chiefly in the posterior column and in the anterior part of the left lateral column. The anterior cornu presents the œdematous appearance already described. The nerve-cells have here undergone extensive vacuolation, clear spherical spaces existing in and outside them as already described.

Tenth dorsal. - The infiltration is chiefly into the middle of the left half of the grey substance, near the 
cervix cornu posterioris; the posterior cornu is also densely infiltrated with cells. These extend along the posterior nerve root, and at the extremity of it are accumulated at the surface of the cord. There is also much infiltration into both posterior columns, and into the anterior part of the right lateral column.

A few sections lower down the infiltration is chiefly into the posterior parts of the two lateral columns, outside the posterior cornua, and it extends to the surface of the cord where are large aggregations of myelin.

Eleventh dorsal nerves. - The infiltration chiefly affects the right half of the cord, involving mainly the peripheral portion of the anterior columns and the posterior part of the lateral columns. A slighter degree of infiltration extends throughout the whole anterior column as far as the grey substance.

About the twelfth dorsal, and between it and the first lumbar nerves, there are enormous aggregations of leucocytes in the right half of the posterior column, and also in both antero-lateral columns. A vessel which passes from the surface into the anterior column is covered with a dense coating of cells exactly similar to those which can be seen here and there within the vessel. Similar cells are infiltrated into the adjacent tissue, but gradually decrease in number away from the vessel. There is also much infiltration of the inner half of the right anterior cornu ; the cells being in places aggregated around vessels, but for the most part scattered uniformly through the grey substance. The large nerve-cells appear very granular. There is also much irregular thickening of the pia mater over the posterior columns.

Second lumbar.-The chief infiltration is in the left anterior column, but there are some dense masses of cells in both anterior cornua, especially in the outer portions. Around one of the vessels which are seen in section the cells form an aggregation about four times the diameter of the vessel. The walls of the vessels appear somewhat thicker than normal. 
Third lumbar nerves. - The central canal is considerably distended, and in its centre is an aggregation of lymphoid cells. The large multipolar nerve-cells appear swollen and granular, with indistinct outlines as already described. The vacuolation is very conspicuous, and in many cells has proceeded to the almost entire destruction of the cell with the exception of the nucleus. Near the cells which appear in a state of disintegration, vessels are seen which are surrounded by lymphoid corpuscles. In the white column, near to the grey matter, are two large oval masses tinting deeply with carmine; in their outer portions round cells are seen resembling in every respect those infiltrated elsewhere; in the centre of the mass, however, only a reticulate structure can be seen, consisting of branching cells which have oval spaces between them. The appearance suggests the idea that these branching cells have developed from such lymphoid cells as are seen on the margin. In the left lateral column a similar change has occurred. There is a considerable increase in the interstitial fibrous tissue, which, in some spots forms, imperfectly fibrous tracts of some size, and in this tissue are many large nuclei resembling the nuclei of the infiltrating cells.

Fourth lumbar nerves.-The round cells are uniformly infiltrated through the left anterior grey cornu, and are in places aggregated around the nerve-cells. The right anterior cornu is in its outer half, and the adjacent part of the lateral column quite destroyed by the invasion of these cells. There is also much destruction in the outer part of the lateral column, where only a few nervefibres can be seen. The rest are blended together into a uniform granular degenerated mass, not tinting with carmine or with logwood. In the inner part of the column the outlines of all the nerve-fibres have disappeared, but the axis-cylinders can still be traced among the cells with which the area is occupied, and which are aggregated very densely along some vessels crossing this part, and just outside the grey substance a vessel so 
covered with leucocytes runs across a clear empty space, which has apparently resulted from the disintegration and destruction of the nervous and connective tissue. The inner half of the posterior cornu and the adjacent external tract of the posterior column is the seat of a dense infiltration, uniform in the corna, but in the white substance distributed in tracts along the vessels. The adjacent fibres of the posterior median column seem to have perished and are replaced by masses of myelin, in which no axis-cylinders can be seen. At the spot thus degenerated there is but little cellular infiltration. A few sections lower down, the infiltration in the posterior cornu becomes much more dense.

A little lower, between the fourth and fifth lumbar nerves, the grey matter presents the same infiltration; there is much less disease in the posterior column, but the interstitial connective tissue is much more abundant than in health, and contains large numbers of minute granule-like nuclei. Almost every nerve-cell in the anterior cornu presents the same granular appearance and indistinct outline already described. Their nuclei and nucleoli remain distinct. A few large nerve-cells near the posterior vesicular column appear much swollen, and their nuclei cannot be seen. The other columns of the cord are fairly healthy.

A few sections lower down there is a wedge-shaped area, having its base to the surface, in which all nerveelements have disappeared, and all that remains is a reticulate connective tissue, consisting of elongated fibres or fibre-cells. In the middle of this tract a vessel runs.

Fifth lumbar nerves.-Infiltration exists in the posterior column and posterior part of the lateral column.

A little lower there is less infiltration, but some exists around the vessels in the anterior columns, and there are a few tracts of cells in the posterior columns. Some nerve-cells appear to be apparently healthy, others appear to be swollen and disintegrating.

A few sections lower down the infiltration of the 
posterior column is very extensive, and tracts extend on each side of, and parallel to, the posterior median fissure, from the surface of the cord almost to the posterior grey commissure, and from them branching tracts of infiltration extend on each side.

In the conus medullaris there is less disease; the most conspicuous change is an infiltration around the vessels in the anterior columns.

Medulla oblongata.-Changes very similar to those found in the spinal cord could be found scattered irregularly through the medulla, the chief change being the aggregation of lymphoid cells in the neighbourhood of vessels, such as has been just described. This change in the medulla was found chiefly in the white columns, and was much less extensive than in the spinal cord.

In the lowest part of the medulla, above the first pair of cervical nerves, the right anterior column was densely infiltrated with cells, some of which surrounded, and here and there concealed, some of the bundles of fibres of the nerve-roots. In the right lateral column were some irregular broad areas, adjacent to the vessels, packed with cells. A few sections higher, the position of a vessel in this column was marked by a large mass of cells, the aggregation being ${ }_{30}$ th of an inch long and ${ }_{150}^{1}$ th broad. It appeared to consist entirely of round lymphoid cells mixed with much granular matter.

The anterior pyramids at and just above the decussation were healthy, but just outside the left pyramid was a slight cellular infiltration. Just above the decussation the left posterior pyramid was the seat of extensive infiltration throughont its entire extent. The right was normal. There was slight infiltration of the right lateral column, and a mass of cells lay in the course of some of the fibres of the left hypoglossal nerve. At the lower portion of the hypoglossal nucleus there was extreme disintegration of the grey matter around the central canal ; the substance was riddled with minute vacuoles separating the nerveelements. Higher up the hypoglossal nucleus was normal. 
In the upper part of the medulla the changes were very slight, being confined to a slight infiltration of the upper portion of the restiform bodies, and a few dilated vessels, occasionally with slight neighbouring cellular infiltration, near the floor of the fourth ventricle.

Cerebellum.-The changes found in the cerebellum were scattered irregularly through all the principal divisions of the organ, so that it was difficult to say that one part was more diseased than another.

The medullary centre presented, scattered through it, alterations similar in character to those which existed in the white substance of the cord. These consisted in the infiltration of the tissue with minute round cells (Plate XIII, fig. 14), for the most part aggregated around the vessels, but in some places scattered through the intervening tissue. In almost every process of medullary substance more or less infiltration could be traced, but the densest change was in the white centre itself, in which here and there tracts of tissue were entirely occupied by this cellular infiltration. The cells were for the most part round, and closely resembled those of the infiltration into the cord, and also those of the adjacent granular layer of the cerebellar cortex. In some, distinct nuclei could seen. Some cells, as in the spinal cord, were angular and fusiform. Where this infiltration was abundant, the nervefibres of the medullary centre were separated by clear spaces, an appearance precisely similar to that seen in some parts of the spinal cord, and which has been spoken of as suggesting a condition of " œdema."

The layers of the cortex were for the most part healthy ; here and there, however, slight changes were apparent. The granule layer was little affected; the only alteration was that in some places the nuclei were separated by clear spaces, or by small collections of yellowish débris, which resisted the carmine. The large corpuscles of Purkinje were for the most part healthy, but in places presented changes similar to those in the large nerve-cells of the cord. They were granular, slightly swollen, surrounded 
by slightly granular débris, and vacuoles existed partly in adjacent, finely-granular material, and partly in the substance of the cells (see Plate XIII, fig. 13). The intervening substance of this layer had in places a degenerated appearance, the tissue elements being separated by minute clear spaces. The external "grey layer," was also for the most part healthy, but here and there was a dilated vessel filled with blood. These were seen especially in the neighbourhood of the change just described in and around the cells of Purkinje. In one place an extravasation of blood had taken place into the most superficial portion of this layer.

CAsE 2.-For an opportunity of examining the spinal cord in the second case we are indebted to Dr. Hughlings Jackson, who has published a note of the symptoms presented by the dog, in the 'Lancet' for May 1 st, 1875.

The movements in this case were confined to one foreleg. Unfortunately we have been unable to learn which fore-leg was affected. The animal was killed by pithing, under chloroform, and after the spinal cord had been divided the animal lived for a few minutes, and during those few minutes the movements in the fore-leg were observed to continue.

An examination in the fresh state revealed no embolism of small arteries, and no obvious disease of the encephalon or spinal cord. The cord and medulla alone were examined with the microscope.

The medulla oblongata was healthy throughout, with the exception of slight damage to the lowest part of the right anterior pyramid, caused apparently by the pithing.

Spinal cord.-The highest portion of the cervical cord was quite healthy. Elsewhere throughout this region the nerve-cells of the anterior cornua were very granular, and appeared somewhat swollen, in some instances so that the body of the cell had an almost globular form. This change in the cells was much more marked in the 
right anterior cornu than in the left, and was almost confined to the middle and lower parts of the cervical enlargement. The granules in some cells were so large and refracting as to appear like spherules of fat (Plate XIII, fig. 12). At the highest part of the cervical enlargement the edge of the anterior column adjacent to the anterior median fissure was for a. short space irregularly infiltrated with minute nuclei, each about $\frac{1}{7000}$ inch in diameter, exactly resembling those normally existing in the neuroglia. In places in the right anterior corna round cells could be seen adjacent to the vessels, apparently an early stage of the cellular infiltration so abundant in the spinal cord just described.

The upper dorsal region of the cord presented nothing abnormal. Below the middle of the dorsal region there was some symmetrical disintegration of the posterior cornua, especially of their outer portions, and at one spot the disintegration extended across the posterior commissure.

In the upper part of the lumbar region the nerve-cells of the right posterior vesicular column were swollen and granular. The difference between the cells of the right and left columns was very marked.

In the mid-lumbar region the left anterior cornu contained some much dilated vessels. A greatly dilated vessel ran along a nerve root through the right anterior column, and outside the vessel was a small extravasation of blood.

The lowest part of the lumbar region presented no abnormality.

\section{Remarks.}

The striking difference in the minute changes found in the two cases renders it difficult to draw any inference as to the morbid condition on which the disease depends. It would appear from the symptoms that the disease in the second case (in which one fore-leg only was effected) was in an earlier and slighter stage 
than in the first case, since in the latter one fore-leg was affected in the first instance, and the spasm afterwards spread to other parts. In the slighter case the only morbid appearance at all conspicuous was the granular and swollen condition of the nerve-cells. The other changes noted were slight and limited in area. This condition of the nerve-cells is one which might well have resulted from such overaction as the clonic spasm necessarily involves. There was evidence of vascular turgescence only here and there. In the other cord a morbid state of the nerve-cells was also apparent, somewhat different from that in the slighter case, but very conspicuous, and also of such a character as may reasonably be ascribed to their overaction. But in this case other extensive lesions were found, consisting of a cellular infiltration related in position to the vessels, and which it seems reasonable to ascribe to a vascular disturbance. This lesion occupied not only the cord but the medulla oblongata and cerebellum, while the corpora striata and hemispheres were free from it.

Two views may be held as to the relation of this cellular infiltration to the disease. It may be regarded as primary, and the cause of the irritation of the nerveelements, or, as secondary to this overaction, to the excessive functional activity of the cerebellum and cord. There are difficulties in the acceptance of either explanation, and the question can probably be decided only by further observations. On the one hand, the distribution of this change was apparently random, conspicuously unsymmetrical, and in no way related to the function of the parts. (In this respect it presents a marked difference from the vascular changes found by Dr. Dickinson in the chorea of man.) From its distribution and extension it seems clear that this cellular infiltration, to whatever cause it may have been due, ran an independent course and produced its own effects. In different places the posterior columns (outer and inner fasciculi), the grey matter (anterior and posterior horns and central part), the 
anterior and lateral white columns, were all affected. The infiltration must have interfered with the function of the structures in which it occurred; in some places, where it was most intense, destruction of the nerve-elements had taken place. The loss of power and the loss of sensation may, with probability, be ascribed to the effect of this local infiltration. On the other hand, the wider limitation of this change to the cerebellum, medulla, and cord, makes it difficult to believe that it can have been due to a primary lesion of the vascular system (such as embolism or thrombosis). Another difficulty in accepting such a view is that we should have to assume a different pathology for the two cases, since in the slighter case no such cellular infiltration or evidence of considerable vascular disturbance was found. It would seem, therefore, most in harmony with the facts of the two cases to assume that in its origin this cellular infiltration depended on a vascular disturbance, which was not primary and independent, but was secondary to the overaction of the nerve-elements. Once set up, the cellular infiltration continued, increased, and produced its own consequences.

It is to be noted that in some places the minute infiltrating cells were becoming angular and fusiform in those parts of the infiltration which appeared of oldest date. This change suggests the probability that the cells may become transformed into connective tissue, and that a condition of disseminated sclerosis may result from this scattered infiltration.

The effect of section of the cord, in the two cases, was singularly at variance. In the one case, in which very slight lesions were found in the cord, the movements persisted after section. This is in accordance with the observations of Chauveau, Onimus, and others, who have found that in similar cases the movements have usually persisted after the section. But in the case in which extensive spinal lesions existed, section of the cord arrested the movements, except in those muscles which are supplied by the medulla oblongata. From this fact 
the inference might be drawn that the movements depended on the encephalic changes, and that the section arrested the transmission of the morbid action. But this conclusion is contradicted by the persistence of the movements after section of the cord in other cases in which the movements resembled closely those in the case in which they ceased. We should have to assume that movements of the same character were, in the one case spinal, in the other encephalic in their origin, and that they were encephalic in the case in which most extensive spinal lesions were found. A more probable explanation of the cessation of the movements is that they were inhibited by the shock of the division of the cord. The nerve-cells may have been weakened by their long-continued overaction, so that their inhibition was more easily produced than in the slighter case in which the disease was in an earlier stage. This is rendered highly probable by an observation of Onimus and Legros, ${ }^{1}$ that in one case the movements ceased for five minutes after section of the cord, and then recommenced.

Other observations will be needed to settle these and some other points of interest which the cases suggest. They show, at any rate, that it is important that the nervecentres should be carefully examined in conditions of disordered nervous action in the lower animals.

I 'Electricité Médicale,' 1872, p. 383. 


\section{DESCRIPTION OF PLATES XII AND XIII.}

The Pathological Anatomy of Canine "Chorea" (W. R. Gowers, M.D., and H. R. O. Sankey).

\section{Plate XII.}

(Case 1.)

Fig. 1. Spinal cord, lumbar enlargement; dense infiltration into right half of anterior grey cornu and adjacent part of lateral column.

Fig. 2. Ditto, lower dorsal; infiltration into left lateral column, and right anterior cornu.

Fig. 3. Upper dorsal; infiltration into both lateral and posterior columns.

Fig. 4. Posterior columns; branching tracts of infiltration.

Fig. 5. Section of vessel with infiltrating cells, round and angular, in vicinity.

Fig. 6. Healthy white column.

Fig. 7. White column, infiltrated with lymphoid cells.

\section{Plate XIII.}

(Cases 1 and 2.)

Figs. 8-11, Morbid appearances of nerve-cells of anterior cornu, spinal cord (8 and 9, vacuolation; and 10 and 11, granular disintegration). Case 1.

Fig. 12. Granular nerve-cell, right anterior cornu of spinal cord, cervical region. Case 2.

Fig. 13. Cerebellum; (Case 1) granular state of cells of Pur. kinje, with vacuoles partly adjacent, partly in protoplasm of cell; distended ressel passing through grey layer and into granule layer.

Fig. 14. Medullary centre of cerebellum; (Case 1) infiltration of cells adjacent to vessel. 
Plate. XII.
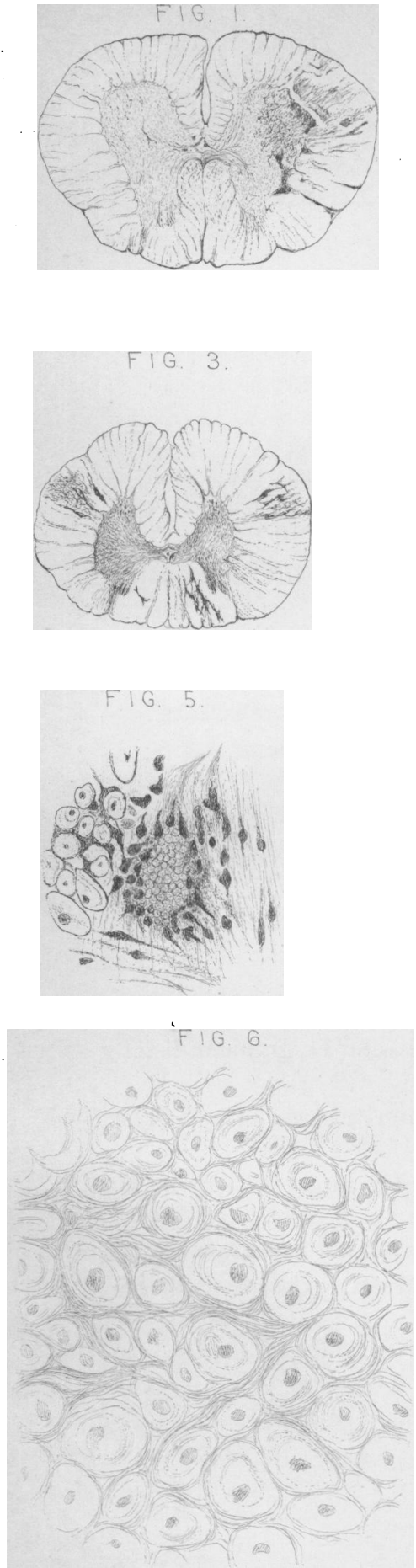

HRO. Sankey de. RVintern Iifh.
Med Chir. Trans. Vol. LX.
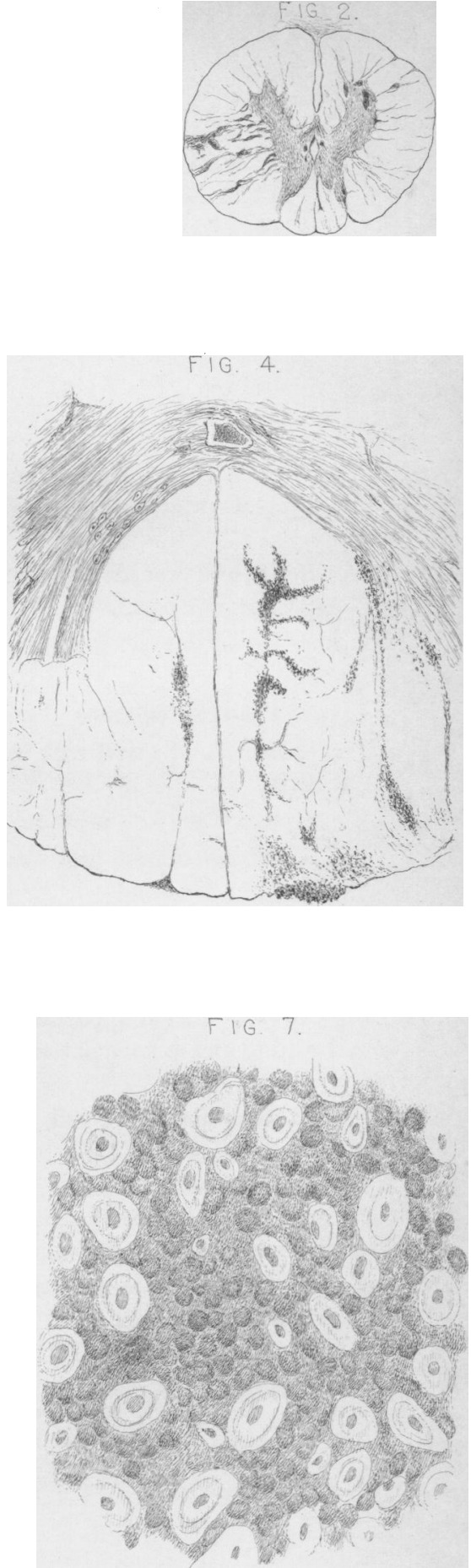

Mintern Bros. imp. 


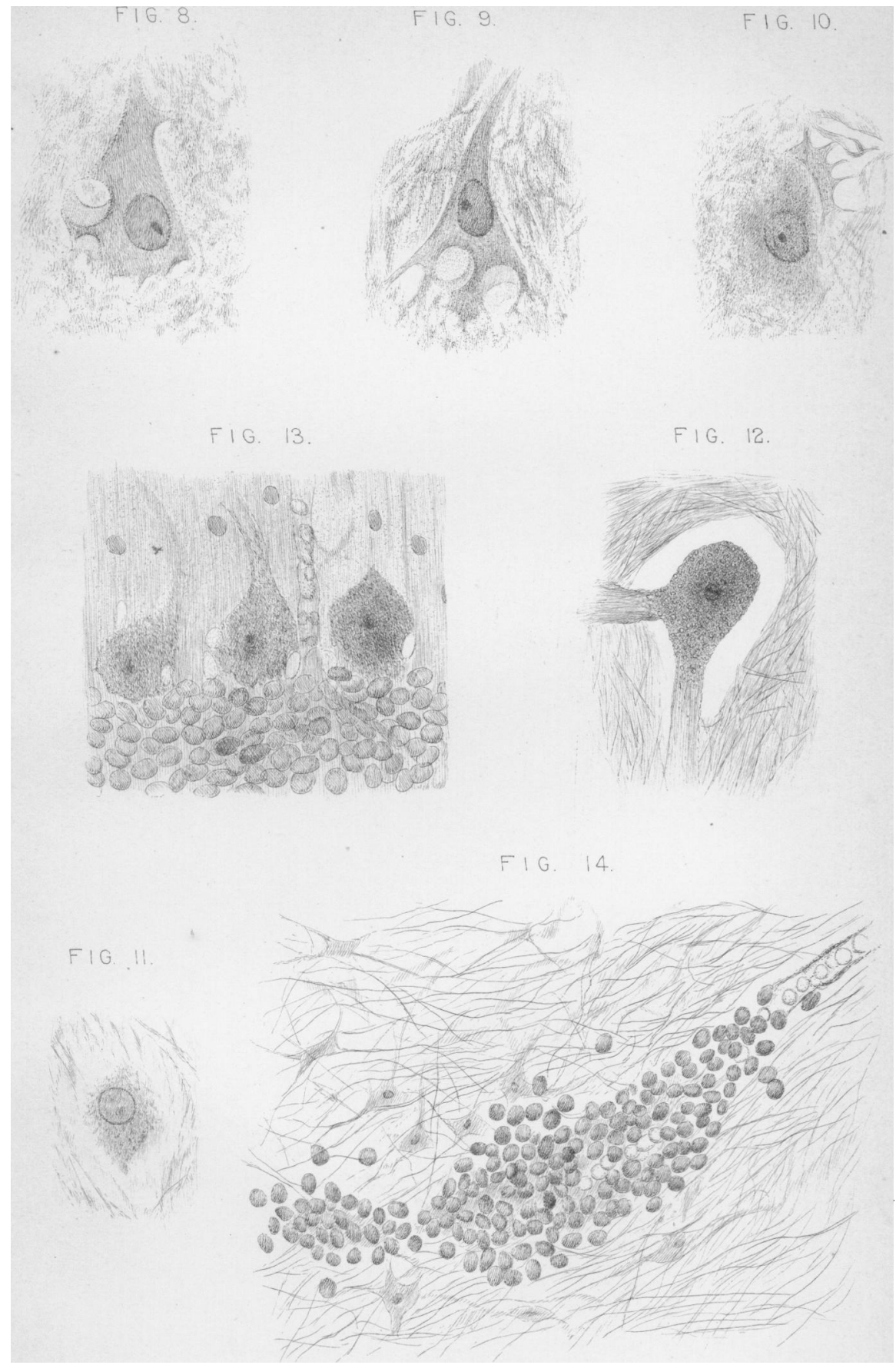

